\title{
PRESTÍGIO ORGANIZACIONAL PERCEBIDO, IDENTIFICAÇÃO ORGANIZACIONAL E EXAUSTÃO EMOCIONAL DO POLICIAL MILITAR
}

\section{PERCEIVED ORGANIZATIONAL PRESTIGE, ORGANIZATIONAL IDENTIFICATION AND EMOTIONAL EXHAUSTION OF MILITARY POLICE OFFICER}

\section{HARRISON BACHION CERIBELI}

Professor Adjunto da Universidade Federal de Ouro Preto (UFOP)

Doutor em Administração - Universidade de São Paulo (FEARP/USP)

Orcid: https://orcid.org/0000-0002-1654-6273

E-mail: harrisonbceribeli@ufop.edu.br

Endereço: Rua do Catete, 166, Centro, Mariana - MG

\section{VIRGÍNIA FILGUEIRAS DE FREITAS}

Graduada em Administração - Universidade Federal de Ouro Preto (UFOP)

Orcid: https://orcid.org/0000-0001-6073-9868

E-mail:vifilgueiras@yahoo.com

\author{
ANA FLÁVIA REZENDE \\ Universidade Federal de Minas Gerais - UFMG \\ Doutoranda em Administração na Universidade Federal de Minas Gerais (UFMG) \\ Orcid: https://orcid.org/0000-0002-1926-0174 \\ E-mail: anaflaviarezendee@gmail.com
}

\section{KARLA LUISA COSTA SABINO}

Professora Assistente da Universidade Federal de Alfenas (UNIFAL)

Doutoranda em Controladoria e Contabilidade - Universidade Federal de Minas Gerais (UFMG)

Orcid: https://orcid.org/0000-0003-3037-4014

E-mail: karla.costa@unifal-mg.edu.br

\begin{abstract}
RESUMO
Como consequência do adoecimento de muitos policiais devido a seu ofício, não apenas eles sofrem, mas também a sociedade. Por isso, torna-se essencial investigar os fatores que contribuem para reduzir o esgotamento do policial militar e, por conseguinte, a incidência de doenças diversas sobre essa categoria profissional. Nesse contexto, definiu-se como objetivo de pesquisa analisar a relação entre o prestígio organizacional percebido, a identificação organizacional e a exaustão emocional do policial militar de Minas Gerais. O método de pesquisa empregado foi a survey e, para analisar os dados coletados, aplicou-se a técnica de Modelagem de Equações Estruturais. Com base nos resultados, concluiu-se que o prestígio organizacional percebido influencia positivamente a identificação organizacional e negativamente a exaustão emocional do agente vinculado à Polícia Militar de Minas Gerais. Ademais, constatou-se que a identificação organizacional exerce uma influência negativa sobre a exaustão emocional do policial militar de Minas Gerais.
\end{abstract}

Palavras-chave : Esgotamento profissional. Exaustão emocional. Prestígio externo percebido. Identificação organizacional. Polícia Militar. 


\section{ABSTRACT}

As a consequence of the illness of many policemen due to their job, not only they suffer, but also the society. Therefore, it is essential to investigate the factors that contribute to reduce the exhaustion of the military police and, consequently, the incidence of diverse diseases on this professional category. In this context, it was defined as a research objective to analyze the relationship between perceived organizational prestige, organizational identification and emotional exhaustion of the military police of Minas Gerais. The research method used was the survey and, to analyze the collected data, the Structural Equation Modeling technique was applied. Based on the results, it was concluded that the perceived organizational prestige positively influences the organizational identification and negatively the emotional exhaustion of the officer linked to the Military Police of Minas Gerais. In addition, it was verified that the organizational identification exerts a negative influence on the emotional exhaustion of the military policeman of Minas Gerais.

Keywords: Professional exhaustion. Emotional exhaustion. Perceived external prestige. Organizational identification. Military Police.

\section{INTRODUÇÃO}

A Polícia Militar de Minas Gerais (PMMG) é considerada a instituição policial mais antiga do Brasil, tendo sua origem em junho de 1775, com a criação do Regimento Regular de Cavalaria de Minas, na cidade de Ouro Preto, que visava garantir o cumprimento da lei e a ordem na sociedade mineira (PMMG, 2018).

A denominação atual, Polícia Militar de Minas Gerais (PMMG), foi admitida a partir de 1946. Antes de receber esta nomenclatura, a instituição era conhecida por outros nomes, como, por exemplo, Corpo Policial Militar, Guarda Republicana, Corpo Militar de Polícia de Minas, Força Policial, Brigada Policial e Força Pública (PMMG, 2018).

Com 243 anos de trabalho no Estado de Minas Gerais, a PMMG guia-se pelos ideais de liberdade e respeito aos direitos humanos e à cidadania, possuindo valores como disciplina, ética, justiça e hierarquia, sendo a disciplina e a hierarquia os pilares das organizações militares (PMMG, 2018).

A instituição tem como objetivos garantir a segurança pública e preservar a ordem pública, resguardando o cidadão, a sociedade e os bens públicos e privados, e trabalha para diminuir a criminalidade e contribuir para a manutenção do bem-estar social (PMMG, 2018).

O trabalho dos policiais militares é considerado uma atividade de alto risco, além de bastante desgastante, devido aos elevados índices brasileiros de criminalidade e às inúmeras situações nas quais eles têm que lidar diretamente com a violência, a tensão e os problemas familiares de terceiros. Ademais, soma-se a esses fatores as pressões causadas pelas rígidas práticas disciplinares de controle adotadas pela instituição (SPODE; MERLO, 2006).

Em decorrência dessas particularidades da profissão, o policial muitas vezes é acometido pela síndrome de Burnout, relacionada ao estresse crônico, que afeta negativamente sua capacidade laboral (COSTA et al., 2007), tornando-lhe, algumas vezes, mais violento com os civis (KOP; EUWEMA; SCHAUFELI, 1999) e mais impulsivo durante o atendimento de uma ocorrência (OLIVEIRA; SANTOS, 2010).

$\mathrm{O}$ estresse persistente provocado por esse ofício pode gerar uma série de danos à saúde física e mental do indivíduo (SOUZA; MINAYO, 2005), que acaba desenvolvendo um quadro de desequilíbrio emocional, tornando-se agressivo no trabalho, emocionalmente 
exausto, mais propício a cometer suicídio e não realizado profissionalmente (OLIVEIRA; SANTOS, 2010).

Complementarmente, como resultado do elevado nível de estresse da profissão, muitos policiais experimentam fadiga constante e sofrem devido a dores musculares, alterações no sono, problemas gastrointestinais, diminuição da atenção e da concentração, baixa autoestima, impaciência, resistência ao relaxar, isolamento e falta de interesse pelo trabalho (OLIVEIRA; SANTOS, 2010). Nos casos em que o estresse crônico gera maiores danos à saúde mental do policial, o sofrimento psíquico tende a dar origem a um quadro de alcoolismo e depressão (LIPP; PEREIRA; SADIR, 2005; SILVA; VIEIRA, 2008).

Diante desse contexto, torna-se evidente a necessidade de investigar como diferentes aspectos podem contribuir para ampliar ou mitigar o esgotamento do policial militar. Seguindo essa linha de investigação, definiu-se como objetivo de pesquisa analisar a relação entre o prestígio organizacional percebido, a identificação organizacional e a exaustão emocional do policial militar do Estado de Minas Gerais.

A justificativa para a realização do presente estudo reside na escassez de trabalhos publicados no Brasil correlacionando os construtos aqui abordados, lacuna que se torna ainda mais severa considerando a realidade dos policiais militares. Além disso, tem-se a expectativa de contribuir com a discussão acerca do que a PMMG pode fazer para reduzir o esgotamento emocional dos policiais, o que, além de favorecer a saúde física e mental desses profissionais, ainda tende a melhorar a qualidade do serviço prestado à sociedade.

\section{REFERENCIAL TEÓRICO}

O referencial teórico deste trabalho foi subdividido em três subtópicos, perpassando pelos principais construtos estudados: prestígio organizacional, identificação organizacional e exaustão emocional do trabalhador.

\subsection{PRESTÍGIO ORGANIZACIONAL}

A reputação que determinada organização possui na sociedade está diretamente vinculada às percepções formadas pelos diferentes agentes sociais; assim, o prestígio organizacional vem do consenso socialmente construído a respeito das características que a organização possui, que podem ser consideradas positivas ou negativas (HIGHHOUSE; LIEVENS; SINAR, 2003).

Sob a ótica dos indivíduos que integram a organização, o prestígio organizacional desdobra-se em duas construções cognitivas: o prestígio externo percebido (PEP) ou prestígio organizacional percebido (POP), que remete à percepção deles acerca de como os não membros a veem, e o respeito interno percebido (RIP), que se refere às percepções que eles próprios, enquanto membros da organização, nutrem sobre ela (TYLER; BLADER, 2003). Verifica-se, portanto, que os indivíduos, além de serem influenciados pela avaliação que fazem da organização onde trabalham, estão expostos e são afetados também pelas opiniões formadas pelas pessoas externas a ela (SMIDTS; PRUYN; VAN RIEL, 2001).

O prestígio externo percebido, que é objeto do presente estudo, está associado à interpretação dos agentes externos à organização acerca de sua atuação e principais características (MAEL; ASHFORTH, 1992; DUTTON; DUKERICH; HARQUAIL, 1994) a partir das opiniões emitidas por grupos sociais influentes (HELM, 2013) e das diversas informações disseminadas sobre ela via comunicação boca a boca e/ou publicidade (SMIDTS; PRUYN; VAN RIEL, 2001), o que reforça o papel das ações de responsabilidade social corporativa (FAROOQ; 
RUPP; FAROOQ, 2017; GÜRLEK; TUNA, 2019; LYTHREATIS; MOSTAFA; WANG, 2019) e dos esforços publicitários que visam melhorar a imagem organizacional perante seus stakeholders (ROSENGREN; BONDESSON, 2017).

Analisando os estudos que abordaram essa temática, verifica-se que a reputação de que frui determinada organização influencia a confiança nela depositada por seus funcionários (NG, 2016), bem como a intenção deles de procurarem outro emprego (HELM, 2013; MISHRA, 2013; RATHI; LEE, 2015; NG, 2016; DENIZ, 2020) e o comprometimento organizacional (OJEDOKUN; IDEMUDIA; DESOUZA, 2015), à medida que estimula o apego emocional dos indivíduos em relação a ela (BRAVO et al., 2017). Ademais, quando a reputação da organização é favorável, os funcionários tendem a se orgulhar por fazerem parte dela (SMIDTS; PRUYN; VAN RIEL, 2001), a experimentar menor exaustão emocional devido ao aumento da identificação organizacional (BAER et al., 2018) e a apresentar maior satisfação no trabalho, passando a manifestar com menor frequência comportamentos contraproducentes durante a execução de suas atividades laborais (TUNA et al., 2016) e a dar referências boca a boca mais positivas sobre ela a terceiros (UEN et al., 2015). Em contrapartida, quando os membros da organização percebem que os não membros alimentam uma imagem desfavorável sobre ela, alguns resultados negativos podem ser observados, tais como maior estresse em decorrência do trabalho, falta de interesse nas atividades laborais e maior rotatividade (DUTTON; DUKERICH; HARQUAIL, 1994). Nessa linha, estabeleceu-se como primeira hipótese de pesquisa que:

$\mathrm{H}_{1}$ : O prestígio organizacional percebido possui relação negativa com a exaustão emocional experimentada pelos policiais militares em seu trabalho.

O pestígio externo percebido também possui relação direta com a identificação organizacional (AKGUNDUZ; BARDAKOGLU, 2017; SU; LEE; LEE, 2019), porquanto os empregados identificam-se mais ou menos com a organização de acordo com a reputação que Ihe atribuem junto à sociedade (FULLER et al., 2006; AL-ATWI; BAKIR, 2014; AKGUNDUZ; BARDAKOGLU, 2017; BRAVO et al., 2017; KLIMCHAK et al., 2019). Ademais, atua como variável mediadora na relação entre a responsabilidade social corporativa e a identificação organizacional (DE ROECK; AKREMI; SWAEN, 2016; FATMA; KHAN; RAHMAN, 2019). Sendo assim, propôs-se como segunda hipótese de pesquisa que:

$\mathrm{H}_{2}$ : O prestígio organizacional percebido possui relação positiva com a identificação organizacional dos policiais militares.

Cabe ponderar, todavia, que quando o prestígio organizacional é elevado, mas o suporte organizacional percebido é baixo, os indivíduos que fazem parte da organização tendem a atribuir a ela maior cinismo organizacional, o que os leva ao desapontamento e ao silêncio quanto aos problemas identificados nos processos de trabalho e possíveis oportunidades de melhorias, evidenciando que a reputação positiva, caso não seja acompanhada por maior suporte aos funcionários, tende a trazer resultados negativos (MIGNONAC et al., 2018). 


\subsection{IDENTIFICAÇÃO ORGANIZACIONAL}

A identificação organizacional (IO) é um construto que foi concebido com base na Teoria da Identidade Social e se refere, portanto, a uma forma específica de identificação social que remete à percepção, formada por um sujeito, de pertencimento à determinada organização (ASHFORTH; MAEL, 1989).

Sob essa ótica, as organizações são interpretadas como categorias sociais mais ou menos capazes de refletirem o autoconceito de seus membros (ASHFORTH; MAEL, 1989) e, neste sentido, existe 10 quando as conviç̧ões do indivíduo relativas a uma organização da qual se é membro tornam-se autorreferências que o auxiliam a definir quem ele é como pessoa (PRATT, 1998).

A identificação organizacional corresponde à extensão do quanto o sujeito se percebe membro da organização da qual faz parte e do quanto essa ligação auxilia-o a se autodefinir (ASHFORTH; MAEL, 1989). Logo, o indivíduo identificado com determinada organização é aquele que vinculou, cognitiva e/ou emocionalmente, o conceito que possui de si mesmo ao conceito que atribui a ela (RIKETTA, 2005).

$\mathrm{Na}$ literatura, são encontrados diferentes fatores que impactam na identificação organizacional, como, por exemplo, a responsabilidade social corporativa percebida pelos empregados (ZHAO; LEE; MOON, 2019), o grau de insegurança que percebem em seu emprego (PICCOLI et al., 2017), a confiança depositada por eles em seus respectivos líderes e a liderança ética, a qual afeta positivamente as experiências dos indivíduos em seu trabalho (DEMIRTAS et al., 2017) e está associada a características como honestidade, respeito e justiça no ambiente laboral (BROWN; TREVIÑO; HARRISON, 2005).

Especificamente em relação à justiça, verifica-se em Asadullah et al. (2017) que as dimensões interpessoal e informacional desse construto influenciam a IO, pois interferem no cumprimento do contrato psicológico pelos líderes da organização. Neste sentido, conclui-se que quando há discrepância entre as expectativas do indivíduo e as práticas de gestão de pessoas adotadas na organização, ou seja, quando há rompimento do contrato psicológico que fora estabelecido, a identificação organizacional tende a ser menor (SMITH et al., 2017).

A IO está associada a um conjunto de atitudes e comportamentos individuais positivos no âmbito do trabalho (RIKETTA, 2005; LEE; PARK; KOO, 2015), como a proatividade demonstrada na execução das atividades laborais (LIU et al., 2018), além de contribuir para alinhar os interesses organizacionais aos do funcionário (HASLAM et al., 2006), pois, à medida que este desenvolve uma identificação mais forte, trabalha com maior comprometimento, visando não apenas o próprio sucesso profissional, mas, de forma concomitante, o alcance dos objetivos da organização (MELEADY; CRISPS, 2017).

De maneira complementar, a 10 estimula as pessoas a nortearem sua atuação de acordo com as necessidades e demandas da organização (PRATT, 2000) e a se tornarem mais leais a seu empregador (ADLER; ADLER, 1988); dessa forma, passam a representá-lo, mesmo que tenham que fazer determinados sacrifícios para isso (ASHFORTH; HARRISON; CORLEY, 2008; RIKETTA, 2005), e a nutrir a intenção de permanecerem vinculados a ele ao longo do tempo (THURSTON; GLENDON, 2018).

Quando os indivíduos desenvolvem uma elevada identificação com a organização, também tendem a experimentar menor tensão no trabalho e, por conseguinte, menor exaustão emocional; por outro lado, a IO, quando atinge níveis muito baixos, passa a agir como um fator estressor que amplia o estresse e o desgaste individuais (CIAMPA et al., 2019). Percebe-se, com isso, que a 10 pode ser estimulada objetivando, entre outros aspectos, a 
redução do esgotamento dos empregados (CARMONA et al., 2006). Neste sentido, delimitouse como terceira hipótese de pesquisa que:

$\mathrm{H}_{3}$ : A identificação organizacional possui relação negativa com a exaustão emocional experimentada pelos policiais militares em seu trabalho.

\subsection{EXAUSTÃO EMOCIONAL}

A exaustão emocional (EE) refere-se a um estado de esgotamento que acomete o trabalhador, estando associada ao estresse e à sobrecarga, física e mental, decorrentes do trabalho realizado (LEE; ASHFORTH, 1996; MASLACH; SCHAUFELI; LEITER, 2001; RUBINO; VOLPONE; AVERY, 2013; SHIH et al., 2013; LI; WONG; KIM, 2017; BAERISWYL et al., 2017).

Tal estado é apontado como a dimensão central ou preponderante da síndrome de Burnout (MASLACH et al., 2001; BAERISWYL et al., 2017), que ainda conta com outras duas dimensões: a baixa realização, que pode ser compreendida como um sentimento de incompetência, e a despersonalização ou indiferença em relação ao trabalho e às pessoas com quem o indivíduo tem contato em decorrência de suas atividades laborais (MASLACH; JACKSON, 1981).

Na síndrome de Burnout, a exaustão reflete os efeitos do estresse experimentado pelo sujeito no trabalho, enquanto a baixa realização e a despersonalização contemplam a forma como ele lida com as demandas profissionais e a sobrecarga, remetendo ao distanciamento cognitivo e emocional no ambiente laboral (MASLACH; SCHAUFELI; LEITER, 2001).

Variados fatores podem levar um indivíduo a experimentar maior exaustão emocional, como, por exemplo, o subemprego, quando se trabalham menos horas do que o desejado (RUBINO; VOLPONE; AVERY, 2013), a sobrecarga de tarefas, as expectativas individuais não atendidas pela organização, os conflitos no trabalho, as exigências elevadas enfrentadas pelo trabalhador no ambiente laboral (MASLACH; SCHAUFELI; LEITER, 2001), as interações estressantes com os supervisores (LEITER; MASLACH, 1988) e o conflito entre trabalho e família (JENSEN, 2014).

De forma complementar, também podem ser apontados como antecedentes da exaustão emocional os relacionamentos conflituosos com colegas de trabalho (SHAUKAT; YOUSAF; SANDERS, 2017), a incivilidade no ambiente laboral (HUANG; LIN, 2017), os conflitos de papeis, a pressão excessiva por resultados (LEE; ASHFORTH, 1996), os baixos níveis de satisfação no trabalho, a ambiguidade associada à função exercida, a falta de autonomia (SHIH et al., 2013) e a vivência, de maneira recorrente, de situações estressantes no ambiente organizacional (TOPPINEM-TANNER; KALIMO; MUTANEN, 2002). Ademais, quando a carga laboral é elevada e o apoio entre os colegas de trabalho é baixo, o indivíduo também tende a experimentar maior esgotamento (BAERISWYL et al., 2017).

A exaustão emocional está relacionada a diferentes aspectos negativos do comportamento humano no trabalho, tais como a intenção de rotatividade e a falta de compromisso organizacional, o que pode ser explicado pelo fato de que o indivíduo, quando se encontra emocionalmente esgotado, passa a ser tomado pelo desejo de fugir das diversas situações que enfrenta em seu dia a dia, apresentando baixo envolvimento com suas atividades laborais e propensão a abandonar a organização (LEE; ASHFORTH, 1996).

Ademais, quando o funcionário apresenta um alto nível de exaustão emocional, sua eficácia fica reduzida (LEITER; MASLACH, 1988) e ele passa a apresentar resistência para ajudar ou cooperar com os demais profissionais da organização, além de não se sentir motivado (BANG; REIO, 2017) e apresentar dificuldades para controlar suas emoções (TANG et al., 
2016), o que prejudica seu desempenho ao executar suas atividades laborais (BANG; REIO, 2017; NIESSEN et al., 2017).

Por fim, a exaustão emocional ainda faz com que os trabalhadores tornem-se menos proativos e propensos a enfrentarem novos desafios, menos engajados com o próprio desenvolvimento (NIESSEN et al., 2017) e menos satisfeitos com seu trabalho (HUTCHINS; PENNEY; SUBLETT, 2018), sentindo-se fatigados ao término de sua jornada laboral (PLUUT et al., 2018). Assim sendo, considerando todas essas consequências perniciosas, fica evidente a necessidade de se investigarem as causas desse estado de esgotamento, com o intuito de se planejarem ações para preveni-lo e mitigá-lo nas organizações contemporâneas.

\section{ASPECTOS METODOLÓGICOS}

Para atingir o objetivo traçado, conduziu-se uma pesquisa descritiva quantitativa: descritiva, à medida que foi delineada com o intuito de se testarem as hipóteses de pesquisa a respeito das relações entre os construtos investigados, e quantitativa, pois foi utilizado um instrumento padronizado para coleta de dados numéricos, empregando-se, posteriormente, técnicas estatísticas para analisá-los.

O método de pesquisa adotado foi a survey, que consiste na coleta de dados a respeito de características, opiniões ou comportamentos de determinado grupo de pessoas por meio da aplicação de um questionário (PINSONNEAULT; KRAEMER, 1993), apresentando como vantagens a possibilidade de acessar um número maior de respondentes em um intervalo menor de tempo e o fato de que são os próprios indivíduos a fonte de informações primárias sobre eles mesmos (HAIR JUNIOR et al., 2005).

Para coleta de dados, elaborou-se um questionário autoadministrado, estruturado com base na escala Likert de sete pontos, sendo nomeados apenas os extremos como "discordo totalmente" e "concordo totalmente". Segundo Camparo (2013), a escala Likert é uma das mais tradicionais em pesquisas científicas que avaliam comportamentos e/ou atitudes, o que se explica pelo fato de que os itens mensurados são relativamente fáceis de serem administrados, quantificados e codificados.

O instrumento de coleta de dados incluiu quatro seções, sendo a primeira constituída por questões demográficas, que subsidiariam a posterior caracterização da amostra, e as três seguintes compostas por questões relativas ao prestígio organizacional percebido, identificação organizacional e exaustão emocional, nesta ordem.

Para mensurar o prestígio organizacional percebido, adaptou-se, para o contexto militar, a escala desenvolvida por Highhouse, Lievens e Sinar (2003), composta por cinco itens. Já para mensurar a identificação organizacional, adotou-se a escala elaborada por Mael e Ashforth (1992), composta por seis itens, a qual também foi adaptada ao contexto militar. Por fim, para mensurar a exaustão emocional, foram empregados nove itens extraídos da escala MBI (Maslach Burnout Inventory) (MASLACH; JACKSON, 1981).

Cabe esclarecer que a escala MBI não foi utilizada integralmente, pois, além de contar com nove itens para mensurar a exaustão emocional, ainda contava com cinco itens para mensurar a despersonalização e oito para mensurar a realização pessoal, que não faziam parte do escopo do presente estudo.

A população-alvo da pesquisa incluiu os policiais militares do Estado de Minas Gerais que estavam na ativa no momento da coleta de dados da pesquisa e que possuíam patentes de soldado, cabo ou sargento. A técnica de amostragem empregada foi a não probabilística por conveniência. De acordo com Curwin e Slater (1991), essa técnica, quando bem aplicada, permite que sejam alcançados resultados satisfatórios de forma rápida e com menor custo. 
Como justificativa para a seleção das unidades amostrais de maneira não aleatória, aponta-se a impossibilidade de se obter uma relação de todos os indivíduos que fazem parte da Polícia Militar de Minas Gerais (PMMG), inviabilizando, por conseguinte, a realização de um sorteio para direcionar a composição da amostra, além de não haver garantia de que os policiais sorteados responderiam ao questionário.

Durante a etapa de coleta de dados da pesquisa, encaminhou-se o questionário elaborado em formato eletrônico, acompanhado de uma breve descrição da proposta do estudo, ao 10 Batalhão da Polícia Militar de Minas Gerais (BPMMG), localizado em Belo Horizonte, e ao 52 BPMMG, localizado em Ouro Preto, sendo, em seguida, distribuído internamente por meio de um sistema de comunicação da corporação. De forma complementar, o link de acesso ao questionário eletrônico foi divulgado, com o auxílio de alguns policiais, em grupos do WhatsApp já existentes, nos quais somente participavam integrantes da PMMG.

Por fim, realizou-se a coleta de dados também de maneira presencial, distribuindo-se uma versão impressa do questionário em duas bases comunitárias móveis da PMMG e no 13을 BPMMG, todos localizados em Belo Horizonte. Cabe acrescentar que tal abordagem somente foi viabilizada depois de se realizar uma visita ao 13을 BMMG e de se efetuar um contato com a Diretoria de Comunicação Organizacional da corporação.

Para definir o tamanho da amostra, seguiram-se as recomendações de Hair Junior et al. (2009) de que deve ser coletado um número de unidades amostrais que represente uma proporção de cinco a 20 vezes a quantidade de variáveis a serem analisadas. Como o presente estudo contou com 20 variáveis, definiu-se que a amostra deveria ser composta por, pelo menos, 300 respondentes, garantindo uma proporção de, no mínimo, 15 unidades amostrais para cada variável de pesquisa.

Para análise dos dados, optou-se pela técnica de Modelagem de Equações Estruturais, que permite que sejam examinadas, de forma simultânea, as relações entre os múltiplos construtos investigados (HAIR JUNIOR et al., 2009). Testou-se um modelo completo de variáveis latentes, que compreende um modelo de mensuração e um modelo estrutural (BYRNE, 2016). O modelo de mensuração retratou o agrupamento das variáveis observadas do estudo em três variáveis latentes, representando os construtos prestígio organizacional percebido (POP), identificação organizacional (IO) e exaustão emocional (EE). Por sua vez, o modelo estrutural descreveu a ligação entre as variáveis latentes criadas, em conformidade com as hipóteses de pesquisa previamente delineadas, sintetizadas na Figura 1.

Figura 1 - Modelo de Caminhos testado

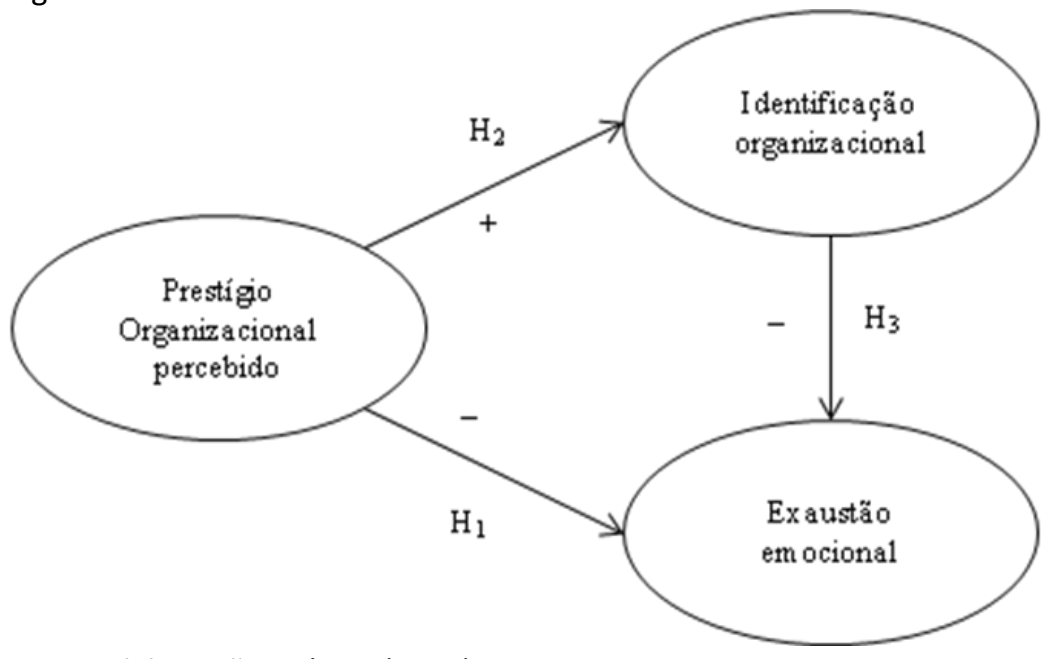

Fonte: Elaboração própria (2018) 


\section{APRESENTAÇÃO E DISCUSSÃO DOS RESULTADOS}

A amostra da pesquisa foi composta por 358 integrantes da Polícia Militar de Minas Gerais, dos quais $86,6 \%$ são do sexo masculino e $13,4 \%$, do sexo feminino. A faixa etária predominante entre os respondentes foi de até 30 anos, que correspondeu a $48,3 \%$ da amostra, seguida pelas faixas etárias de 31 a 40 anos, 41 a 50 anos e 50 anos ou mais, que representaram $33 \%, 16,8 \%$ e $1,9 \%$ das unidades amostrais, respectivamente.

Ainda em relação às características da amostra, os policiais militares casados e solteiros representaram, respectivamente, $52,2 \%$ e $40,5 \%$ dos respondentes, enquanto $7,3 \%$ informaram outros estados civis. Por fim, quanto à graduação ou patente, as unidades amostrais encontram-se distribuídas da seguinte forma: Soldados $1^{\text {a }}$ Classe $(46,4 \%)$, Cabos $(23,7 \%)$, Terceiros-sargentos $(15,4 \%)$, Segundos-sargentos (10,6\%), Primeiros-sargentos $(2,8 \%)$ e Soldados 2 a Classe $(1,1 \%)$.

Finda a caracterização da amostra, realizou-se uma análise preliminar com o intuito de verificar a normalidade dos dados coletados, utilizando-se, para isso, duas medidas: a assimetria e a curtose, cujos valores devem estar contidos dentro do intervalo entre $-1,96$ e $+1,96$ (HAIR JUNIOR et al., 2009). O que se observou, todavia, é que a maioria das variáveis apresentou assimetria e/ou curtose com valor que extrapolava os limites intervalares estabelecidos. Constatou-se, portanto, que as variáveis da pesquisa não seguem uma distribuição normal.

Em seguida, calculou-se o Alfa de Cronbach para verificar a consistência interna de cada um dos conjuntos de variáveis utilizados para mensurar os construtos da pesquisa (CARVALHO; CASSIANI, 2012). Como parâmetro, os valores obtidos devem ser superiores a $0,60 \mathrm{ou}$, adotando um padrão mais conservador, 0,70 (HAIR JUNIOR et al., 2009). Efetuados os cálculos, foram obtidos coeficientes de 0,939, 0,882 e 0,931 para os grupos de variáveis relacionados, respectivamente, aos construtos prestígio organizacional percebido, identificação organizacional e exaustão emocional, evidenciando uma elevada confiabilidade das escalas empregadas. Com isso, confirmou-se a possibilidade de agrupar as variáveis originais do estudo.

Posteriormente, utilizando o software AMOS, empregou-se, para análise dos dados, a técnica de Modelagem de Equações Estruturais. Adotou-se, em razão da não-normalidade dos dados que fora inicialmente constatada, o método de estimação assintoticamente livre de distribuição, que se mostra insensível à violação do pressuposto de normalidade (HAIR JUNIOR et al., 2009).

O primeiro modelo testado possuía 20 variáveis observadas, sendo cinco relacionadas ao prestígio organizacional percebido, seis, à identificação organizacional, e nove, à exaustão emocional. Com o intuito de validá-lo, analisaram-se as seguintes medidas de ajuste: índice de qualidade de ajuste calibrado (AGFI), que deve ser igual ou superior a 0,8 (RHEE; ULEMAN; LEE, 1996; STACCIARINI; PACE, 2017); índice de qualidade de ajuste (GFI), que deve estar próximo ou ser superior a 0,9 (SCHUMACKER; LOMAX, 2010); raiz do erro quadrático médio de aproximação (RMSEA), cujo valor deve estar dentro do intervalo de 0,05 a 0,08 (SCHUMACKER; LOMAX, 2010); e qui-quadrado normado ( $\chi^{2}$ normado), que deve ser inferior a 5,0 (HAIR JUNIOR et al., 2009). Como os índices obtidos indicaram qualidade de ajuste pobre $\left(A G F I=0,854 ; G F I=0,884 ;\right.$ RMSEA $=0,09$; e $\chi^{2}$ normado $\left.=3,868\right)$, optou-se pela exclusão de algumas variáveis, utilizando-se as cargas fatoriais como parâmetro.

Com isso, foram excluídas da análise as variáveis IDO1 (Quando alguém critica a PMMG, parece um insulto pessoal) e IDO6 (Se uma reportagem na mídia criticasse a PMMG, eu me sentiria envergonhado), cujas cargas fatoriais foram de 0,289 e 0,332, respectivamente, 
enquanto seria recomendado que fossem superiores a 0,5 para que obtivessem significância prática (HAIR JUNIOR et al., 2009).

Em seguida, rodou-se um segundo modelo, que contou com 18 variáveis observadas, sendo cinco relacionadas ao prestígio organizacional percebido, quatro, à identificação organizacional, e nove, à exaustão emocional. Para esse modelo, os índices encontrados foram: $\mathrm{AGFI}=0,857 ; \mathrm{GFI}=0,889 ; \mathrm{RMSEA}=0,083$; e $\chi^{2}$ normado $=3,482$. Como a qualidade de ajuste ainda se manteve em um patamar insatisfatório, foram necessárias novas rodadas, excluindo-se, uma a uma, as variáveis que apresentaram as menores cargas fatoriais.

Para a terceira rodada, excluiu-se a variável EXA7 (Eu sinto que estou trabalhando muito); para a quarta rodada, excluiu-se a variável EXA6 (Eu me sinto frustrado pelo meu trabalho); finalmente, para a quinta rodada, excluiu-se a variável IDO2 (Eu me interesso pelo que as pessoas pensam a respeito da PMMG).

No quinto modelo testado, que contou com 15 variáveis observadas, os índices obtidos mostraram-se adequados conforme a literatura consultada: $\mathrm{AGFI}=0,861 ; \mathrm{GFI}=0,899 ; \mathrm{RMSEA}$ $=0,079 ; \mathrm{e} \chi^{2}$ normado $=3,203$. Encontrou-se, portanto, uma solução satisfatória, que pode ser observada na Figura 2.

Figura 2 - Modelo validado

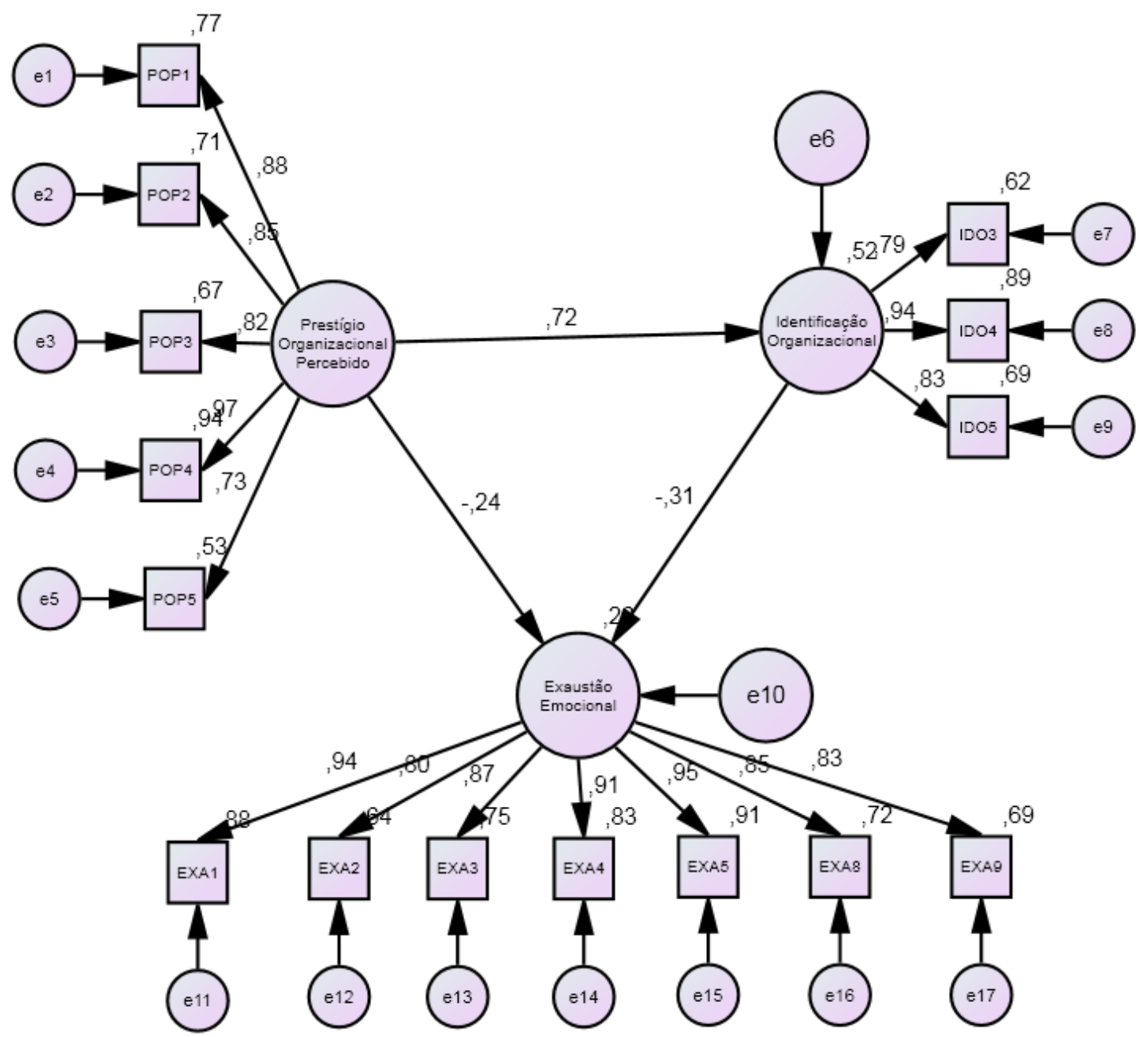

Fonte: Elaboração própria no software AMOS (2018).

Em seguida, para ratificar as três variáveis latentes criadas a partir do agrupamento das variáveis observadas, calcularam-se a variância média extraída e a confiabilidade composta, cujos valores devem ser superiores a, respectivamente, 0,5 e 0,7 (HAIR JUNIOR et 
al., 2009). Os resultados obtidos, sintetizados no Quadro 1 , indicaram que as variáveis latentes geradas representam, de maneira adequada, as variáveis observadas que thes deram origem.

Quadro 1 - Valores referentes à variância média extraída e confiabilidade composta

\begin{tabular}{|c|c|c|}
\hline Variável latente & Variável média extraída & Confiabilidade composta \\
\hline Prestígio organizacional percebido & 0,7244 & 0,9918 \\
\hline Identificação organizacional & 0,7351 & 0,9886 \\
\hline Exaustão emocional & 0,7756 & 0,9967 \\
\hline
\end{tabular}

Fonte: Elaboração própria (2018)

Complementando os cálculos anteriores, realizou-se uma análise dos p-valores referentes às relações entre as variáveis observadas e as respectivas variáveis latentes nas quais elas foram agrupadas, adotando-se um nível de $95 \%$ de confiança. Como todos os pvalores obtidos foram inferiores a 0,001 , pode-se afirmar que os agrupamentos propostos são estatisticamente significativos. É possível validar, portanto, o modelo de mensuração delineado, cuja estrutura final foi sintetizada no Quadro 2.

Quadro 2 - Estrutura final do modelo de mensuração

\begin{tabular}{|c|c|}
\hline \multirow{5}{*}{$\begin{array}{c}\text { Prestígio } \\
\text { Organizacional } \\
\text { Percebido }\end{array}$} & $\begin{array}{l}\text { POP1: Os militares provavelmente se orgulham em dizer que trabalham na } \\
\text { PMMG. }\end{array}$ \\
\hline & POP2: A PMMG é uma organização respeitável para se trabalhar. \\
\hline & $\begin{array}{l}\text { POP3: A PMMG provavelmente possui a reputação de ser uma excelente } \\
\text { empregadora. }\end{array}$ \\
\hline & POP4: Eu acho que a PMMG é um lugar de prestígio para se trabalhar. \\
\hline & POP5: Provavelmente há muitas pessoas que gostariam de trabalhar na PMMG. \\
\hline \multirow{3}{*}{$\begin{array}{l}\text { Identificação } \\
\text { Organizacional }\end{array}$} & IDO3: Quando falo sobre a PMMG, costumo dizer "nós" ao invés de "eles". \\
\hline & IDO4: Considero os sucessos da PMMG como meus sucessos. \\
\hline & IDO5: Quando alguém elogia a PMMG, parece um elogio pessoal. \\
\hline \multirow{7}{*}{$\begin{array}{l}\text { Exaustão } \\
\text { Emocional }\end{array}$} & EXA1: Eu me sinto emocionalmente esgotado (a) no meu trabalho. \\
\hline & EXA2: Eu me sinto esgotado(a) no final de um dia de trabalho. \\
\hline & $\begin{array}{l}\text { EXA3: Eu me sinto fatigado(a) quando me levanto de manhã e tenho que } \\
\text { enfrentar outro dia no trabalho. }\end{array}$ \\
\hline & EXA4: Trabalhar com pessoas o dia todo é realmente desgastante para mim. \\
\hline & EXA5: Eu me sinto esgotado por causa do meu trabalho. \\
\hline & EXA8: Trabalhar diretamente com pessoas causa-me estresse. \\
\hline & EXA9: Sinto-me no limite de minhas possibilidades. \\
\hline
\end{tabular}

Fonte: Elaboração própria (2018)

Posteriormente, prosseguiu-se para a análise do modelo estrutural, com o intuito de se testarem as relações entre as variáveis latentes endógenas (IO e EE) e exógena (POP) criadas para representar os construtos investigados na pesquisa.

A primeira relação analisada foi entre os construtos prestígio organizacional percebido e identificação organizacional, que apresentou um p-valor de 0,000 e, portanto, mostrou-se estatisticamente significativa. Como o beta obtido foi de 0,723 , conclui-se que se trata de uma relação diretamente proporcional, ou seja, quanto maior o prestígio da PMMG percebido pelos policiais militares, maior a identificação que eles desenvolvem em relação à corporação. Logo, confirmou-se $\mathrm{H}_{2}$.

A segunda relação examinada foi entre os construtos identificação organizacional e exaustão emocional, que apresentou p-valor de 0,000 e beta de -0,310, ou seja, trata-se de uma relação estatisticamente significativa e inversamente proporcional. Logo, pode-se 
afirmar que, quanto maior a identificação dos policiais militares em relação à PMMG, menor sua exaustão emocional decorrente do trabalho, o que permitiu confirmar $\mathrm{H}_{3}$.

Por fim, a terceira e última relação analisada foi entre os construtos prestígio organizacional percebido e exaustão emocional, que obteve p-valor de 0,000 e beta de -0,241. Trata-se, portanto, de uma relação estatisticamente significativa e inversamente proporcional, ou seja, quanto maior o prestígio da PMMG percebido pelos policiais militares, menor a exaustão emocional experimentada por eles em decorrência de suas atividades laborais. Tal resultado possibilitou a ratificação de $\mathrm{H}_{1}$.

Concluída a análise estatística dos dados coletados, faz-se necessário conduzir uma reflexão, com base na literatura previamente consultada, a respeito dos resultados encontrados, iniciando-se pela confirmação da relação entre o POP e a 10 , que encontra respaldo nos estudos de Klimchak et al. (2019), Su, Lee e Lee (2019), Akgunduz e Bardakoglu (2017), Bravo et al. (2017), Al-Atwi e Bakir (2014) e Fuller et al. (2006). Para isso, deve-se, preliminarmente, resgatar a definição de identificação organizacional, que remete a um estado no qual o indivíduo vinculou cognitiva e/ou emocionalmente seu autoconceito ao conceito que atribui à determinada organização da qual é membro (RIKETTA, 2005). Neste sentido, assumindo que o indivíduo identificado com a organização é aquele que passa a se autodefinir com base na ligação que possui com ela (ASHFORTH; MAEL, 1989; PRATT, 1998), compreende-se como natural a tendência observada de o policial militar mineiro desenvolver uma maior identificação com a PMMG quando percebe que ela é bem avaliada pela sociedade e frui de uma reputação positiva, já que, neste caso, ele passa a nutrir maior orgulho por fazer parte da corporação e, por conseguinte, a valorizar mais seu vínculo com ela, o que, com o tempo, faz com que ele incorpore a sua autodefinição o fato de pertencer a essa instituição.

Quanto à relação corroborada entre a $\mathrm{IO}$ e a EE, seguindo na mesma linha conceitual, depreende-se que o indivíduo identificado com a organização tende a experimentar menor tensão no trabalho e, por conseguinte, menor exaustão emocional devido ao fato de considerar seu vínculo com ela como parte de quem ele é como pessoa (CIAMPA et al., 2019; CARMONA et al., 2006). Neste sentido, o policial militar mineiro que se identifica mais com a PMMG experimenta menor esgotamento por ter naturalizado para si mesmo seu status de membro da corporação, passando a se sentir efetivamente como parte dela (ASHFORTH; MAEL, 1989) e, com isso, contando com mais recursos emocionais para lidar com os conflitos e/ou as dificuldades diversas relacionadas à profissão. Da mesma maneira, quando o policial militar mineiro não se sente parte da PMMG, a tendência é que experimente maior esgotamento, porquanto, neste caso, sua avaliação pessoal de que "não pertence a esse lugar" torna-se para ele um fator estressor que leva a um maior desgaste em decorrência de suas atividades laborais.

Analisando conjuntamente essas duas primeiras relações corroboradas empiricamente, conclui-se que a identificação organizacional desempenha o papel de variável mediadora na relação entre o prestígio organizacional percebido e a exaustão emocional, o que encontra respaldo no estudo de Baer et al. (2018). Neste sentido, à medida que o policial militar avalia que a reputação da PMMG é positiva perante a sociedade, mais fortemente ele incorpora a seu autoconceito seu vínculo com a instituição, passando a se sentir efetivamente parte dela e, em decorrência disso, vivenciando uma menor tensão no trabalho, o que contribui para que ele não se desgaste emocionalmente em demasia devido às demandas provenientes de suas atividades laborais.

Além da relação indireta entre o POP e a EE, mediada pela $I O$, também se confirmou que existe uma relação direta entre esses dois construtos. Logo, quanto mais positiva é a 
percepção dos policiais militares mineiros a respeito da reputação da PMMG, menos eles tendem a se desgastar emocionalmente em seu trabalho, o que se justifica ao se considerar que o prestígio organizacional percebido aumenta a confiança depositada na organização por seu público interno (NG, 2016), assim como o orgulho (SMIDTS; PRUYN; VAN RIEL, 2001) e a satisfação dos membros por fazerem parte dela (TUNA et al., 2016), à medida que estimula o apego emocional deles em relação a ela (BRAVO et al., 2017). Seguindo nessa linha, infere-se que a satisfação, a confiança e o orgulho dos policiais militares, provenientes da reputação positiva que atribuem à corporação, funcionam como fatores que suavizam o impacto de eventuais tensões e/ou conflitos oriundos do trabalho, reduzindo a possibilidade de eles atingirem um estado de exaustão emocional.

Conclui-se, portanto, que uma das linhas de ação que a PMMG pode adotar para mitigar o esgotamento dos policiais e, com isso, reduzir o adoecimento físico e mental desses profissionais (OLIVEIRA; SANTOS, 2010; SOUZA; MINAYO, 2005) é direcionar esforços para melhorar sua reputação perante a sociedade, o que pode ser feito combinando programas de responsabilidade social corporativa (FAROOQ; RUPP; FAROOQ, 2017; GÜRLEK; TUNA, 2019; LYTHREATIS; MOSTAFA; WANG, 2019) e ações publicitárias (ROSENGREN; BONDESSON, 2017).

Os programas de responsabilidade social corporativa são importantes, porque tendem a influenciar de maneira positiva a construção de uma imagem favorável para a organização junto a seus stakeholders (DE ROECK; AKREMI; SWAEN, 2016; FATMA; KHAN; RAHMAN, 2019). No caso da PMMG, projetos como o PROERD (Programa Educacional de Resistência às Drogas), que a aproximam da comunidade, devem ser estimulados. Ademais, a instituição poderia ampliar seu envolvimento em campanhas de doação de sangue e de arrecadação de alimentos e agasalhos para atender as pessoas mais vulneráveis socialmente. Do mesmo modo, poderia ainda promover campanhas de arrecadação de brinquedos e/ou itens geriátricos, realizando, subsequentemente, visitas aos locais destinados ao acolhimento infantil e/ou de idosos para distribuição do que foi arrecadado. Finalmente, seria desejável que promovesse eventos esportivos e/ou concursos culturais voltados para jovens e crianças, construindo um vínculo com esses públicos específicos. Obviamente, todas essas propostas tratam-se de exemplos de ações que poderiam ser adotadas, não constituindo, de forma alguma, uma listagem exaustiva das possibilidades de atuação nessa área.

Cabe acrescentar que, ao seguir nessa linha, a PMMG não pode menosprezar a necessidade de comunicar adequadamente as ações e projetos de responsabilidade social que estão sendo realizados, não com o intuito de se autopromover para obter projeção política, mas sim para sinalizar à comunidade que a corporação está se esforçando para participar mais ativamente de seu dia a dia e colaborar para aumentar seu bem-estar.

Ainda com relação à comunicação, poder-se-ia implantar um programa voltado para aumentar a transparência das ações da PMMG, divulgando para a sociedade os resultados alcançados (p. e. recuperação de itens furtados e roubados), para que as pessoas passem a ter uma visão mais clara a respeito da contribuição da instituição.

Por fim, visando melhorar a reputação da PMMG, faz-se necessário investir na capacitação dos policiais que lidam diretamente com o público, para que as interações com a comunidade sejam permeadas por gentileza e solicitude, pois não adiantaria executar uma série de projetos sociais se as pessoas que procuram a instituição forem recebidas com frieza. É importante que a comunidade não apenas veja os esforços da corporação para melhorar o bem-estar de todos, mas também se sinta abraçada quando necessitar da PMMG. Conforme os resultados obtidos indicaram, isso também tende a beneficiar os policiais militares 
mineiros, que, além de se identificarem mais com a corporação, sentir-se-ão menos esgotados.

\section{CONSIDERAÇÕES FINAIS}

A Polícia Militar de Minas Gerais exerce uma importante função, porque visa proteger os cidadãos, garantir a ordem pública e contribuir para a manutenção do bem-estar social. Mas, para que esses objetivos de fato sejam alcançados, os policiais precisam usufruir de boa saúde física e mental. Todavia, o que se percebe é que, na atualidade, muitos desses profissionais estão adoecendo devido a seu ofício. E, em decorrência disso, não apenas eles sofrem, mas também a sociedade.

Nesse contexto, torna-se relevante investigar os diferentes fatores que podem contribuir para que o policial militar experimente menor esgotamento e, consequentemente, adoeça menos. Com isso, definiu-se como objetivo de pesquisa analisar a relação entre o prestígio organizacional percebido, a identificação organizacional e a exaustão emocional do policial militar do Estado de Minas Gerais.

A partir dos resultados obtidos, verificou-se que os policiais militares que atribuem maior prestígio à corporação, ou seja, que consideram que a Polícia Militar de Minas Gerais é valorizada no meio em que está inserida, identificam-se mais com ela e ainda experimentam menor esgotamento emocional no trabalho. Além disso, constatou-se que, à medida que os policiais militares desenvolvem maior identificação com a PMMG, ficam menos desgastados devido a suas atividades laborais.

Conclui-se, portanto, que o prestígio organizacional percebido e a identificação organizacional são importantes ferramentas para mitigar a exaustão emocional dos policiais militares do Estado de Minas Gerais. Logo, para que esses profissionais se desgastem menos durante o trabalho, é importante que a PMMG invista em ações para melhorar a imagem que a sociedade nutre em relação a ela.

Nessa linha, sugere-se que sejam implantadas mais ações de cunho social voltadas para aproximar a Polícia Militar das comunidades mineiras e que a instituição ofereça treinamentos constantes para preparar psicologicamente o policial que tem contato com os civis, para que as interações realizadas sejam amistosas e pautadas na solicitude.

A presente pesquisa traz duas contribuições relevantes: em primeiro lugar, auxilia a preencher uma lacuna na literatura acerca de como diferentes aspectos relacionados ao universo laboral interagem entre si e afetam a exaustão emocional do trabalhador; além disso, estimula a reflexão a respeito de algumas linhas de ação que podem ser exploradas pela PMMG para que os policiais experimentem menor desgaste e, por conseguinte, adoeçam menos, prestando um serviço cada vez melhor para a população mineira.

Para finalizar, recomenda-se que sejam investigados, em estudos futuros, outros aspectos que tenham o potencial de contribuir para que a exaustão do policial militar seja reduzida, tais como o suporte organizacional percebido e o suporte do supervisor percebido, e que o mesmo modelo conceitual aqui proposto seja testado junto a policiais de outros Estados brasileiros, de modo que os resultados obtidos possam ser confrontados e validados.

Complementarmente, sugere-se que sejam realizados estudos de caso de batalhões da Polícia Militar que conseguiram melhorar seu prestígio perante a comunidade junto a qual atuam, com o intuito de se construir um arcabouço de boas práticas que possa orientar os esforços globais dessa instituição voltados para aperfeiçoar sua imagem, reduzindo, dessa forma, o esgotamento emocional dos policiais. 


\section{REFERÊNCIAS}

ADLER, P. A; ADLER, P. Intense loyalty in organizations: a case study of College Athletics. Administrative Science Quarterly, v. 33, n. 3, p. 401-417, 1988. DOI: https://doi.org/10.2307/2392716

AKGUNDUZ, Y.; BARDAKOGLU, O. The impacts of perceived organizational prestige and organization identification on turnover intention: the mediating effect of psychological empowerment. Current Issues in Tourism v. 20, n. 14, p. 1510-1526, 2017. DOI: https://doi.org/10.1080/13683500.2015.1034094

AL-ATWI, A. A.; BAKIR, A. Relationships between status judgments, identification, and counterproductive behavior. Journal of Managerial Psychology, v. 29, n. 5, p. 472-489, 2014. DOI: https://doi.org/10.1108/JMP-02-2012-0040

ASADULLAH, M. A; AKRAM, A; IMRAN, H; ARAIN, G. A. When and which employees feel obliged: a personality perspective of how organizational identification develops. Journal of Work and Organizational Psychology, v. 33, p. 125-135, 2017. DOI:

https://doi.org/10.1016/j.rpto.2017.02.002

ASHFORTH, B. E; MAEL, F. Social identity theory and the organization. Academy of Management Review, v. 14, p. 20-39, 1989. DOI: https://doi.org/10.2307/258189

ASHFORTH, B. E; HARRISON, S., H; CORLEY, K. G. Identification in organizations: an examination of four fundamental questions. Journal of Management, v. 34, p. 325-374, 2008. DOI: https://doi.org/10.1177/0149206308316059

BAER, M. D.; BUNDY, J.; GARUD, N.; KIM, J. K. The benefits and burdens of organizational reputation for employee well-being: a conservation of resources approach. Personnel Psychology, v. 71, n. 4, p. 571-595, 2018. DOI: https://doi.org/10.1111/peps.12276

BAERISWYL, S; KRAUSE, A; ELFERING, A; BERSET, M. How workload and coworker support relate to emotional exhaustion: the mediating role of sickness presenteeism. International Journal of Stress Management, v. 24, n. 1, p. 52-73, 2017. DOI: https://doi.org/10.1037/str0000018

BANG, H; REIO, T. G. Examining the role of cynicism in the relationships between burnout and employee behavior. Revista de Psicologia del Trabajo y de las Organizaciones, v. 33, n. 3, p. 217-227, 2017. DOI: https://doi.org/10.1016/j.rpto.2017.07.002

BRAVO, R.; BUIL, I.; CHERNATONV, L.; MARTÍNEZ, E. Brand identity management and corporate social responsibility: an analysis from employees' perspective in the banking sector. Journal of Business Economics and Management, v. 18, n. 2, p. 241-257, 2017. DOI: https://doi.org/10.3846/16111699.2016.1209785 
BROWN, M. E; TREVIÑO, L. K; HARRISON, D. A. Ethical leadership: A social learning perspective for construct development and testing. Organizational Behavior and Human Decision Processes, v. 97, p. 117-134, 2005.

DOI: https://doi.org/10.1016/j.obhdp.2005.03.002

BYRNE, B.M. Structural Equation Modeling with Amos: basic concepts, applications, and programming. 3. ed. New York: Routledge, 2016.

CAMPARO, J. A geometrical approach to the ordinal data of Likert scaling and attitude measurements: the density matrix in psychology. Journal of Mathematical Psychology, $v$. 57, n. 1-2, p. 29-42, 2013. DOI: https://doi.org/10.1016/j.jmp.2013.02.004

CARMONA, C; BUUNK, A. P; PEIRO, J. M; RODRIGUES, I; BRAVO, M. J. Do social comparison and coping styles play a role in the development of Burnout? Cross-Sectional and longitudinal findings. Journal of Occupational and Organizational Psychology, v. 79, n. 1, p. 85-100, 2006. DOI: https://doi.org/10.1348/096317905X40808

CARVALHO, R. E. F. L; CASSIANI, S. H. B. Questionário atitudes de segurança: adaptação transcultural do Safety Attitudes Questionnaire - Short Form 2006 para o Brasil. Revista Latino-Americana de Enfermagem, v. 20, n. 3, p. 575-582, 2012.

DOI: https://doi.org/10.1590/S0104-11692012000300020

CIAMPA, V.; STEFFENS, N. K.; SCHUH, S. C.; FRACCAROLI, F.; VAN DICK, R. Identity and stress: an application of the expanded model of organisational identification in predicting strain at work. Work \& Stress, v. 33, n. 4, p. 351-365, 2019.

DOI: https://doi.org/10.1080/02678373.2018.1521884

COSTA, M; ACCIOLY JR., H; OLIVEIRA, J; MAIA, E. Estresse: diagnóstico dos policiais militares em uma cidade brasileira. Revista Panamericana de Salud Pública, v. 21, n. 4, p. 217-22, 2007.

CURWIN, J; SLATER, R. Quantitative methods for business decisions. 3. ed. Stamford: Cengage Learning Business Press, 1991.

DE ROECK, K.; AKREMI, A. E.; SWAEN, V. Consistency matters! How and when does corporate social responsibility affect employees' organizational identification? Journal of Management Studies, v. 53, n. 7, p. 1141-1168, 2016. DOI: https://doi.org/10.1111/joms.12216

DENIZ, S. The relationship between perception of corporate reputation and turnover intention: results from Turkey. Journal of Health Management, p. 1-11, 2020. DOI: https://doi.org/10.1177/0972063420909200

DEMIRTAS, O; HANNAH, S. T; GOK, K; ARSLAN, A; CAPAR, N. The moderated influence of ethical leadership, via meaningful work, on followers' engagement, organizational identification, and envy. Journal of Business Ethics, v. 145, n. 1, p. 183-199, 2017. DOI: https://doi.org/10.1007/s10551-015-2907-7 
DUTTON, J. E.; DUKERICH, J. M.; HARQUAIL, C. V. Organizational images and member identification. Administrative Science Quarterly, v. 39, n. 2, p. 239-263, 1994. DOI: https://doi.org/10.2307/2393235

FAROOQ, O; RUPP, D. E; FAROOQ, M. The multiple pathways through which internal and external corporate social responsibility influence organizational identification and multifoci outcomes: the moderating role of cultural and social orientations. Academy of Management Journal, v. 60, n. 3, p. 954-985, 2017. DOI: https://doi.org/10.5465/amj.2014.0849

FATMA, M.; KHAN, I.; RAHMAN, Z. Striving for legitimacy through CSR: an exploration of employee's responses in controversial industry sector. Social Responsibility Journal, v. 15, n. 7, p. 924-938, 2019. DOI: https://doi.org/10.1108/SRJ-07-2017-0116

FULLER, J.; HESTER, K.; BARNETT, T.; FREY, L.; RELYEA, C.; BLEU, D. Perceived external prestige and internal respect: new insights into the organizational identification process. Human Relations, v. 59, n. 6, p. 815-846, 2006. DOI: https://doi.org/10.1177/0018726706067148

GÜRLEK, M.; TUNA, M. Corporate social responsibility and work engagement: evidence from the hotel industry. Tourism Management Perspectives, v. 31, p. 195-208, 2019. DOI: https://doi.org/10.1016/j.tmp.2019.05.004

HAIR JUNIOR, J. F.; BABIN, B; MONEY, A. H; SAMOUEL, P. Fundamentos de métodos de pesquisa em Administração. Porto Alegre: Bookman, 2005.

HAIR JUNIOR, J. F.; BLACK, W. C; BABIN, B. J; ANDERSON, R. E; TATHAM, R. L. Análise multivariada de dados. Porto Alegre: Bookman, 2009.

HASLAM, S. A; RYAN, M. K; POSTMES, T; SPEARS, R; JETTEN, J; WEBLEY, P. Sticking to our guns: Social identity as a basis for the maintenance of commitment to faltering organizational projects. Journal of Organizational Behavior, v. 27, p. 607-628, 2006. DOI: https://doi.org/10.1002/job.370

HELM, S. A matter of reputation and pride: associations between perceived external reputation, pride in membership, job satisfaction and turnover intentions. British Journal of Management, v. 24, p. 542-556, 2013. DOI: https://doi.org/10.1111/j.14678551.2012.00827.x

HIGHHOUSE, S.; LIEVENS, F.; SINAR, E. F. Measuring attraction to organizations. Educational and Psychological Measurement, v. 63, p. 986-1001, 2003. DOI:

https://doi.org/10.1177/0013164403258403

HUANG, H. T.; LIN, C. P. Assessing ethical efficacy, workplace incivility, and turnover intention: a moderated-mediation model. Review of Managerial Science, p. 1-24, 2017. DOI: https://doi.org/10.1007/s11846-017-0240-5 
HUTCHINS, H. M; PENNEY, L. M; SUBLETT, L. W. What imposters risk at work: exploring imposter phenomenon, stress coping, and job outcomes. Human Resource Development Quarterly, v. 29, n. 1, p. 31-48, 2018. DOI: https://doi.org/10.1002/hrdq.21304

JENSEN, M. T. Exploring business travel with work-family conflict and the emotional exhaustion component of burnout as outcome variables: the job demands-resources perspective. European Journal of Work and Organizational Psychology, v. 23, n. 4, p. $497-$ 510, 2014. DOI: https://doi.org/10.1080/1359432X.2013.787183

KLIMCHAK, M.; WARD, A.-K.; MATTHEWS, M.; ROBBINS, K.; ZHANG, H. When does what other people think matter? The influence of age on the motivators of organizational identification. Journal of Business and Psychology, v. 34, p. 879-891, 2019. DOI: https://doi.org/10.1007/s10869-018-9601-6

KOP, N; EUWEMA, M; SCHAUFELI, W. Burnout, job stress, and violent behavior among Dutch police officers. Work \& Stress, v. 13, n. 4, p. 326-340, 1999.

DOI: https://doi.org/10.1080/02678379950019789

LEE, R. T; ASHFORTH, B. E. A meta-analytic examination of the correlates of the three dimensions of job Burnout. Journal of Applied Psychology, v. 81, n. 2, p. 123-133, 1996. DOI: https://doi.org/10.1037//0021-9010.81.2.123

LEE, E.-S.; PARK, T.-Y.; KOO, B. Identifying organizational identification as a basis for attitudes and behaviors: a meta-analytic review. Psychological Bulletin, v. 141, n. 5, p. 1049-1080, 2015. DOI: https://doi.org/10.1037/bul0000012

LEITER, M. P; MASLACH, C. The impact of interpersonal environment on Burnout and organizational commitment. Journal of Organizational Behavior, v. 9, n. 4, p. 297-308, 1988. DOI: https://doi.org/10.1002/job.4030090402

LI, J; WONG, I. A; KIM, W. G. Does mindfulness reduce emotional exhaustion? A multilevel analysis of emotional labor among casino employees. International Journal of Hospitality Management, v. 64, p. 21-30, 2017. DOI: https://doi.org/10.1016/j.ijhm.2017.03.008

LIPP, M. N.; PEREIRA, M. B.; SADIR, M. A. Crenças irracionais como fontes internas de stress emocional. Revista Brasileira de Terapias Cognitivas, v. 1, n. 1, p. 29-34, 2005.

LIU, Y; LI, R; ZHOU, L; TIAN, F. The relationship between organizational identification and internal whistle-blowing: the joint moderating effects of perceived ethical climate and proactive personality. Review of Managerial Science, v. 12, p. 113-134, 2018. DOI: https://doi.org/10.1007/s11846-016-0214-z

LYTHREATIS, S.; MOSTAFA, A. M. S.; WANG, X. Participative leadership and organizational identification in SMEs in the MENA Region: testing the roles of CSR perceptions and pride in membership. Journal of Business Ethics, v. 156, p. 635-650, 2019. DOI: 10.1007/s10551-017$3557-8$ 
MAEL, F.; ASHFORTH, B. E. Alumni and their alma mater: a partial test of the reformulated model of organizational identification. Journal of Organizational Behavior, v. 13, p. 103-123, 1992. DOI: https://doi.org/10.1002/job.4030130202

MASLACH, C; JACKSON, S. E. The measurement of experienced burnout. Journal of Organizational Behavior, v. 2, n. 2, p. 99-113, 1981.

DOI: https://doi.org/10.1002/job.4030020205

MASLACH, C; SCHAUFELI, W. B; LEITER, M. P. Job burnout. Annual Review of Psychology, v. 52, p. 397-422, 2001. DOI: https://doi.org/10.1146/annurev.psych.52.1.397

MELEADY, R; CRIPS, R. J. Take it to the top: imagined interactions with leaders elevates organizational identification. The Leadership Quarterly, v. 28, p. 621-638, 2017. DOI: https://doi.org/10.1016/j.leaqua.2017.01.008

MIGNONAC, K.; HERRBACH, O.; ARCHIMI, C. S.; MANVILLE, C. Navigating ambivalence: perceived organizational prestige-support discrepancy and its relation to employee cynicism and silence. Journal of Management Studies, v. 55, n. 5, p. 837-872, 2018. DOI:

https://doi.org/10.1111/joms.12330

MISHRA, S. K. Perceived external prestige and employee outcomes: mediation effect of organizational identification. Corporate Reputation Review, v. 16, n. 3, p. 220-233, 2013. DOI: https://doi.org/10.1057/crr.2013.9

NG, M. Examining social exchange among Chinese service workers: the mediating effect of trust in organization. Asia Pacific Journal of Business Administration, v. 8, n. 2, p. 163-176, 2016. DOI: https://doi.org/10.1108/APJBA-12-2015-0102

NIESSEN, C; MADER, I; STRIDE, C; JIMMIESON, N. L. Thriving when exhausted: the role of perceived transformational leadership. Journal of Vocational Behavior, v. 103, p. 41-51, 2017. DOI: https://doi.org/10.1016/j.jvb.2017.07.012

OJEDOKUN, O.; IDEMUDIA, E. S.; DESOUZA, M. Perceived external prestige as a mediator between quality of work life and organisational commitment of public sector employees in Ghana. SA Journal of Industrial Psychology, v. 41, n. 1, p. 1-10, 2015. DOI:

http://dx.doi.org/10.4102/sajip.v41i1.1216

OLIVEIRA, K. L; SANTOS, L. M. Percepção da saúde mental em policiais militares da força tática e de rua. Sociologias, n. 25, p. 224-250, 2010. DOI: https://doi.org/10.1590/S151745222010000300009

PICCOLI, B.; CALLEA, A.; URBINI, F.; CHIRUMBOLO, A.; INGUSCI, E.; DE WITTE, H. Job insecurity and performance: the mediating role of organizational identification. Personnel Review, v. 46, n. 8, p. 1508-1522, 2017. DOI: https://doi.org/10.1108/PR-05-2016-0120 
PINSONNEAULT, A; KRAEMER, K. L. Survey research methodology in management information systems: an assessment. Journal of Management Information System, v. 10, n. 2, p. 75-105, 1993. DOI: https://doi.org/10.1080/07421222.1993.11518001

PLUUT, H; ILIES, R; CURSEU, P. L; LIU, Y. Social support at work and at home: dual-buffering effects in the work-family conflict process. Organizational Behavior and Human Decision Processes, v. 146, p. 1-13, 2018. DOI: https://doi.org/10.1016/j.obhdp.2018.02.001

POLÍCIA MILITAR DE MINAS GERAIS - PMMG. Identidade Organizacional. Disponível em: < https://www.policiamilitar.mg.gov.br/portal-

$\mathrm{pm} /$ portalinstitucional/conteudo.action? conteudo=2156\&tipoConteudo=itemMenu $>$. Acesso em: 21 junho 2018.

PRATT, M.G. To be or not to be? Central questions in organizational identification. In: WHETTEN, D.; GODFREY, P. (Eds.). Identity in organizations: building theory through conversations. Thousand Oaks: Sage, 1998. p. 171-207.

PRATT, M. G. The good, the bad, and the ambivalent: managing identification among Amway Distributors. Administrative Science Quarterly, v. 45, n. 3, p. 456-493, 2000. DOI: https://doi.org/10.2307/2667106

RATHI, N; LEE, K. Retaining talent by enhancing organizational prestige: an HRM strategy for employees working in the retail sector. Personnel Review, v. 44, n. 4, p. 454-469, 2015. DOI: https://doi.org/10.1108/PR-05-2013-0070

RHEE, E; ULEMAN, J. S; LEE, H. K. Variations in collectivism and individualism by ingroup and culture: Confirmatory Factor Analysis. Journal of Personality and Social Psychology, v. 71, n. 5, p. 1037-1054, 1996. DOI: https://doi.org/10.1037/0022-3514.71.5.1037

RIKETTA, M. Organizational identification: a meta-analysis. Journal of Vocational Behavior, v. 66, n. 2, p. 358-384, 2005. DOI: https://doi.org/10.1016/j.jvb.2004.05.005

ROSENGREN, S; BONDESSON, N. How organizational identification among retail employees is affected by advertising. Journal of Retailing and Consumer Services, v. 38, p. 204-209, 2017. DOI: https://doi.org/10.1016/j.jretconser.2017.06.005

RUBINO, C; VOLPONE, S. D; AVERY, D. R. Burnout on Mars and Venus: exploring gender differences in emotional exhaustion. Gender in Management: An International Journal, v. 28, n. 2, p.74-93, 2013. DOI: https://doi.org/10.1108/17542411311303220

SCHUMACKER, R. E.; LOMAX, R. G. A beginner's guide to Structural Equation Modeling. 3. ed. New York: Routledge, 2010.

SHAUKAT, R.; YOUSAF, A.; SANDERS, K. Examining the linkages between relationship conflict, performance and turnover intentions: role of job burnout as a mediator. International Journal of Conflict Management, v. 28, n. 1, p. 4-23, 2017.

DOI: https://doi.org/10.1108/IJCMA-08-2015-0051 
SHIH, S.; JIANG, J. J; KLEIN, G; WANG, E. Job Burnout of the information technology worker: work exhaustion, depersonalization, and personal accomplishment. Information \& Management, v. 50, p. 582-589, 2013. DOI: https://doi.org/10.1016/j.im.2013.08.003

SILVA, M. B; VIEIRA, S. B. O processo de trabalho do militar estadual e a saúde mental. Saúde e Sociedade, v. 17, n. 4, p. 161-170, 2008. DOI: http://dx.doi.org/10.1590/S010412902008000400016

SMIDTS, A.; PRUYN, A. T. H.; VAN RIEL, C. B. The impact of employee communication and perceived external image on organizational identification. Academy of Management Journal, v. 44, n. 5, p. 1051-1062, 2001. DOI: http://dx.doi.org/10.2307/3069448

SMITH, L. G. G; GILLESPIE, N; CALLAN, V. J; FITZSIMMONS, T. W; PAULSEN, N. Injunctive and descriptive logics during newcomer socialization: the impact on organizational identification, trustworthiness, and self-efficacy. Journal of Organizational Behavior, v. 38, p. 487-511, 2017. DOI: https://doi.org/10.1002/job.2131

SOUZA, E. R.; MINAYO, M. C. S. Policial, risco como profissão: morbimortalidade vinculada ao trabalho. Ciência e Saúde Coletiva, v. 10, n. 4, p. 917-928, 2005. DOI:

https://doi.org/10.1590/S1413-81232005000400015

SPODE, C.; MERLO, A. R. C. Trabalho policial e saúde mental: uma pesquisa junto aos capitães da Polícia Militar. Psicologia: Reflexão e Crítica, v. 19, n. 3, p. 362-370, 2006. DOI: https://doi.org/10.1590/S0102-79722006000300004

STACCIARINI, T. S. G.; PACE, A. E. Análise fatorial confirmatória da escala: Appraisal of Selfcare Agency Scale - Revised. Revista Latino-Americana de Enfermagem, v. 25, p. 1-9, 2017. DOI: https://doi.org/10.1590/1518-8345.1378.2856

SU, C.-L.; LEE, E. M.; LEE, Y.-D. An empirical research of environment management strategy: exploring the relationships among perceived corporate social responsibility, organizational trust, perceived external prestige and organizational identification. International Journal of Organizational Innovation, v. 12, n. 1, p. 245-260, 2019.

TANG, G; KWAN, H. K; ZHANG, D; ZHU, Z. Work-family effects of servant leadership: the roles of emotional exhaustion and personal learning. Journal of Business Ethics, $v$. 137, n. 2, p. 285-297, 2016. DOI: https://doi.org/10.1007/s10551-015-2559-7

THURSTON, E.; GLENDON, I. Association of risk exposure, organizational identification, and empowerment, with safety participation, intention to quit, and absenteeism. Safety Science, v. 105, p. 212-221, 2018. DOI: https://doi.org/10.1016/j.ssci.2018.02.012

TOPPINEN-TANNER, S; KALIMO, R; MUTANEN, P. The process of Burnout in white-collar and blue-collar jobs: eight-year prospective study of exhaustion. Journal of Organizational Behavior, v. 23, p. 555-570, 2002. DOI: https://doi.org/10.1002/job.155 
TUNA, M.; GHAZZAWI, I.; YESILTAS, M.; TUNA, A. A.; ARSLAN, S. The effects of the perceived external prestige of the organization on employee deviant workplace behavior: the mediating role of job satisfaction. International Journal of Contemporary Hospitality Management, v. 28, n. 2, p. 366-396, 2016. DOI: https://doi.org/10.1108/IJCHM-04-20140182

TYLER, T. R.; BLADER, S. L. The group engagement model: procedural justice, social identity, and cooperative behavior. Personality and Social Psychology Review, v. 7, n. 4, p. 349-361, 2003. DOI: https://doi.org/10.1207/S15327957PSPR0704_07

UEN, J. F.; AHLSTROM, D.; CHEN, S.; LIU, J. Employer brand management, organizational prestige and employees' word-of-mouth referrals in Taiwan. Asia Pacific Journal of Human Resources, v. 53, n. 1, p. 104-123, 2015. DOI: https://doi.org/10.1111/1744-7941.12024

ZHAO, L.; LEE, J.; MOON, S. Employee response to CSR in China: the moderating effect of collectivism. Personnel Review, v. 48, n. 3, p. 839-863, 2019. DOI:

https://doi.org/10.1108/PR-05-2017-0146 